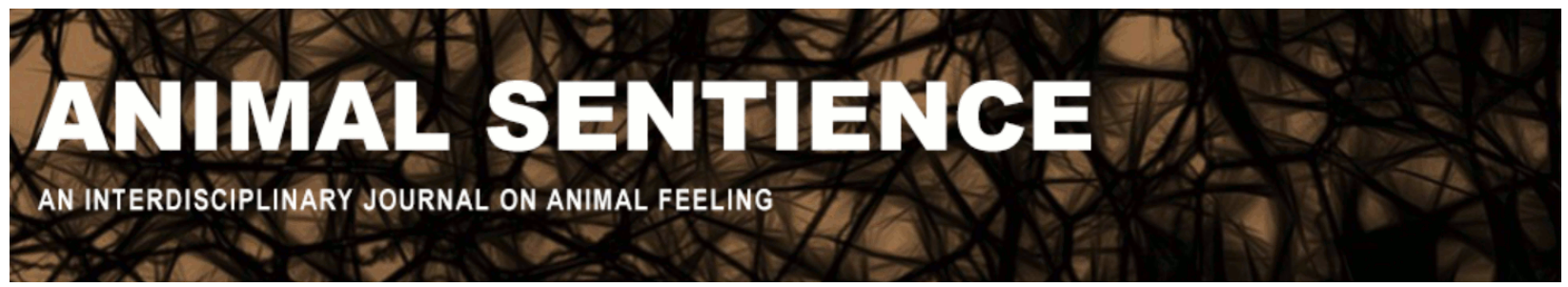

Walters, Edgar T. (2016) Pain-capable neural substrates may be widely available in the animal kingdom. Animal Sentience 3(37)

DOI: $10.51291 / 2377-7478.1067$

Date of submission: $2015-11-24$

Date of acceptance: 2015-12-13

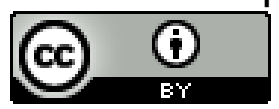

This article has appeared in the journal Animal

Sentience, a peer-reviewed journal on animal

cognition and feeling. It has been made open access,

free for all, by WellBeing International and deposited

in the WBI Studies Repository. For more information,

please contact

wbisr-info@wellbeingintl.org.

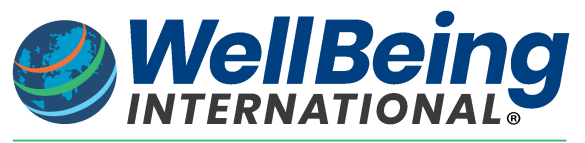

SOLUTIONS FOR PEOPLE, ANIMALS AND ENVIRONMENT 


\title{
Pain-capable neural substrates may be widely available in the animal kingdom
}

\author{
Commentary on Key on Fish Pain
}

\author{
Edgar T. Walters \\ Department of Integrative Biology and Pharmacology \\ University of Texas Medical School at Houston
}

\begin{abstract}
Neural and behavioral evidence from diverse species indicates that some forms of pain may be generated by coordinated activity in networks far smaller than the cortical pain matrix in mammals. Studies on responses to injury in squid suggest that simplification of the circuitry necessary for conscious pain might be achieved by restricting awareness to very limited information about a noxious event, possibly only to the fact that injury has occurred, ignoring information that is much less important for survival, such as the location of the injury. Some of the neural properties proposed to be critical for conscious pain in mammals are also found in the nervous systems of numerous species, including invertebrates. These considerations suggest that simple forms of pain might be present in some animals that lack large brains and complex behaviors.
\end{abstract}

Edgar T. Walters Edgar.T.Walters@uth.tmc.edu is Professor of Integrative Biology and Pharmacology and holder of the Fondren Chair in Cellular Signaling at the University of Texas Medical School at Houston. His research has focused on longterm alterations of neural and behavioral responses to peripheral injury in molluscs (Aplysia and squid), on mechanisms of chronic pain induced by spinal cord injury in rodents, and on understanding the evolution of pain-related mechanisms. https://med.uth.edu/ibp/faculty/edgar-t-walters/

Arguments about which species can and cannot feel pain are often based on behavioral observations and philosophical assumptions. In contrast, Key (2016) argues that fish (and by implication many other animals) do not feel pain because they lack neural substrates equivalent to the cortical pain matrix in mammals. There is now overwhelming evidence that neocortical processing is important for human pain experience, as reviewed by Key, and that cortical processing makes major contributions to pain-related behavior in rodent preclinical models (e.g., Barthas et al., 2015; Koga et al., 2015). Key asserts that pain is experienced in the cerebral cortex and, following influential theories about mechanisms of consciousness, he proposes that long-range binding of gamma oscillations across the cortical pain matrix is a core mechanism for the conscious experience of pain. Key acknowledges that it is not known how synchronized rhythmic activity of widespread populations of neurons actually generates a feeling such as pain, but he is confident that neural network properties essential for these cortical oscillations are required. The two properties he considers most important are signal amplification and global integration, which, Key asserts, are lacking in the nociceptive networks of fish. 
Key's rejoinder to several criticisms of this thesis is to note that the critics fail to provide mechanistic alternatives to cortical processing for generating conscious pain in fish. I will argue that fish, like many other "lower" animals, possess neural networks with properties equivalent to many of those posited by Key as essential for the conscious experience of pain. Let me begin by agreeing with Key that fish do not experience pain in the same way that humans do. Pain scientists have demonstrated that human pain comes in countless varieties and is highly dependent upon a person's past experience, current context, and expectations of the future. In fish, information processing, including nociceptive information processing, is undoubtedly much more limited than in humans - a difference predicted by the much smaller brains and restricted behavioral repertoires of fish. On the other hand, Key's claim is that fish do not feel pain, not that fish do not feel human pain. The central question is whether fish lack the capacity for conscious experience of any kind of pain.

Most pain scientists accept the definition of the International Association for the Study of Pain that pain is "an unpleasant sensory and emotional experience associated with actual or potential tissue damage...." By this definition, pain requires conscious, unpleasant awareness of actual or potential injury (noxious stimulation), but pain does not require awareness of the many features typically associated with human pain, including the location, duration, and severity of noxious stimulation, or closely associated feelings (anxiety, depression) linked to experience with injury and expectations about its consequences. The neocortical features Key deems important for pain are specializations that evolved for highly efficient processing of enormous amounts of information by cognitively advanced mammals, and which certainly facilitate cognitive integration and representation of the numerous properties he lists for human pain ("attention, discrimination, localization, duration, intensity, unpleasantness, valence, and motivational value"). However, conscious awareness of noxious stimulation can, in principle, be mediated by much simpler neural networks than the mammalian cortical pain matrix if much less information is involved (simpler network inputs) and if less flexibility is needed in generating adaptive behavioral responses (simpler network outputs).

Simpler forms of consciousness would correspond to the "raw experience" or "primitive feelings" dismissed by Key because of his assumption that fish lack an adequate neural substrate for any kind of consciousness. However, it is plausible that consciousness of only the valence of an animal's physiological state could be biologically useful, and this type of passive awareness should require much less neural computational power than the types of active, information-rich awareness familiar to humans. Evidence for how little information might be sufficient for nociceptive awareness comes from another free-swimming aquatic organism, the squid, which has a lifestyle remarkably similar to fish. Squid exhibit striking sensitization of defensive responses following injury to an arm or fin (Crook et al., 2011), and this nociceptive sensitization has been shown to enhance the survival of the injured animals (Crook et al., 2014). As in mammals, this behavioral sensitization is paralleled by enhanced sensitivity and activity in sensory neurons that encode noxious stimuli (nociceptors). Surprisingly, and quite unlike mammals, the long-lasting hyperactivity of squid nociceptors after focal injury is not restricted to those innervating an injured fin. Instead, hyperactivity is quite general, occurring in nociceptors on both sides of the body (Crook et al., 2013). This suggests that the location on the 
body of an injury is relatively unimportant to squid, which like fish (and unlike mammals) have little or no ability to tend to a wound or selectively protect it.

On the other hand, the simple fact that a squid has received a significant injury (one potentially noticeable to a predator) is extremely important, resulting in a major change in behavioral state that has significant costs and benefits. An intriguing question is whether the spontaneous activity in widespread squid nociceptors resulting from focal injury has a role like Key's cortical "signal amplification" in driving a neural network that allows a squid to be conscious of the valence of its physiological state; that is, that it has been injured. In this case, the signal amplification would be generated by a generalized activation of peripheral nociceptors, rather than recurrent excitation within a specialized central network, but it might have a similar outcome in driving coherent network activity from which some kind of awareness emerges. In both squid and fish, simple awareness of the fact of an injury sufficiently severe to attract predatory interest may be far more important for minimizing further risk than conscious attention to details of the injury. Awareness of no more than the valence of the altered bodily state (the aversiveness of injury) would make this a form of pain.

On a related note, Key suggests that a lack of apparent effects on normal behavior (feeding, swimming, mating) of fish after injuries such as crude craniotomy indicate an absence of pain. In free-swimming organisms that lack safe refuges, individuals that fail to disguise their compromised condition are magnets for attacks by predators or conspecifics. Alteration of overt behavior is easily detected, especially in shoaling organisms, so aquatic animals that cannot hide are under strong selection pressures to continue their activities as normally as possible after injury. This does not mean that their internal state is unchanged, or that they are not in some sense aware of their more vulnerable condition. Except for the initial escape behavior during injury, human observers saw no difference in the overt behavior of injured and uninjured squid after experimental injury to an arm. The resulting hypervigilant state was recognized only when a predator approached, which evoked much earlier and stronger defensive responses (Crook et al., 2014).

Defensive motivational states triggered by noxious stimulation, which may or may not affect spontaneous behavior, probably occur in many animals. For example, after Aplysia (large, sluglike marine snails) received a neutral chemical stimulus paired with strong noxious stimulation, they later displayed no obvious response to the chemical stimulus alone. However, when test stimuli that elicit defensive or appetitive responses were then applied in the presence of the chemical stimulus, the Aplysia showed dramatic enhancement of various defensive responses and suppression of feeding behavior (Walters et al., 1981). In mammals, such conditioned motivational states resulting from noxious experience are felt as learned fear. This and various other reports - some from other commentators in this issue - indicate that complex motivational states are linked to noxious experience in "lower" animals.

The widespread occurrence of such states raises interesting questions about the species and types of neural organization that are most likely to manifest some kind of conscious awareness in pain-like and fear-like states. Behavioral tests of the aversiveness of internal states produced 
by noxious stimulation will be particularly important for addressing these questions. Powerful operant methods, notably conditioned place preference and conditioned place aversion tests, are available but have rarely been used in studies of pain-like states in non-mammalian vertebrates or in invertebrates. Indeed, these useful tests are quite uncommon in the very large field of preclinical pain research in mammals, which has largely relied on simple reflexive measures that fail to capture the aversive component of pain. With rare exceptions (e.g., Barthas et al., 2015), preclinical studies of the cortical pain matrix have depended upon these limited reflexive indicators of pain.

What neural substrates could mediate simple consciousness of noxious stimulation? As with any form of consciousness, we can only speculate, but it is interesting to use Key's functional arguments as a starting point. Key considers signal amplification and global integration important because he assumes along with various theorists that consciousness somehow emerges from the coordinated activity of large numbers of neurons. In humans, signal amplification leads to widespread neural activity, while global integration helps the extended network synchronize, shape, and sustain this activity. As argued above, simple consciousness of injury should require substantially fewer neurons than the information-rich consciousness possible in mammalian brains. Do mechanisms exist in simpler nervous systems that enable the coordinated activity of a substantial fraction of neurons following noxious stimulation?

Basic mechanisms of neural network activation and integration are highly conserved, and those mentioned by Key as important for conscious pain (extensive, synchronous activation of relevant neuronal populations, local inhibitory circuits, reciprocal excitatory and inhibitory interconnections among these neuronal populations) have been found in nearly all nervous systems that have been examined neurophysiologically, including invertebrate nervous systems. Detection of bodily injury and the adaptive generation and integration of overt as well as internal state responses to injury have a very high biological priority in most animals (Walters, 1994), so it seems likely in these species that many neurons will be activated concurrently during significant noxious stimulation.

The number of neurons intensely and coordinately activated during noxious stimulation may be relatively large even in simple brains. For example, optical imaging of the largest aggregation of neurons in Aplysia (found in each pedal ganglion) revealed that virtually all of the recordable neurons showed long-lasting responses to stimulation of a peripheral nerve at an intensity that activated nociceptors (Bruno et al., 2015). While the recorded population included motor neurons, and the authors interpreted their results in terms of motor program generation, most of the pedal ganglion neurons had unknown functions. Regardless of how many of the recorded neurons contributed to nociceptive information processing, this example of massively synchronized, complex activity triggered by noxious stimulation illustrates the potential of relatively simple nervous systems to generate patterns of activity that in some species might be linked to simple forms of pain.

The logical possibility that coordinated activity of perhaps hundreds or thousands of neurons (rather than many millions) may produce an aversive awareness of noxious stimulation 
reinforces the opinion that fish, amphibians, reptiles, and some invertebrates may experience forms of conscious pain. Nonetheless, we still know very little about when and why during evolution some neural mechanisms became capable of generating pain.

\section{References}

Barthas, F., Sellmeijer, J., Hugel, S., Waltisperger, E., Barrot, M., and Yalcin, I. (2015). The anterior cingulate cortex is a critical hub for pain-induced depression. Biol Psychiatry 77, 236-245.

Bruno, A. M., Frost, W. N., and Humphries, M. D. (2015). Modular deconstruction reveals the dynamical and physical building blocks of a locomotion motor program. Neuron 86, 304-318.

Crook, R. J., Dickson, K., Hanlon, R. T., and Walters, E. T. (2014). Nociceptive sensitization reduces predation risk. Curr Biol 24, 1121-1125.

Crook, R. J., Hanlon, R. T., and Walters, E. T. (2013). Squid have nociceptors that display widespread long-term sensitization and spontaneous activity after bodily injury. $J$ Neurosci 33, 10021-10026.

Crook, R. J., Lewis, T., Hanlon, R. T., and Walters, E. T. (2011). Peripheral injury induces longterm sensitization of defensive responses to visual and tactile stimuli in the squid Loligo pealeii, Lesueur 1821. J Exp Biol 214, 3173-3185.

Key, B. (2016). Why fish do not feel pain. Animal Sentience 2016.003.

Koga, K., Descalzi, G., Chen, T., Ko, H. G., Lu, J., Li, S., Son, J., Kim, T., Kwak, C., Huganir, R. L., Zhao, M. G., Kaang, B. K., Collingridge, G. L., and Zhuo, M. (2015). Coexistence of two forms of LTP in ACC provides a synaptic mechanism for the interactions between anxiety and chronic pain. Neuron 85, 377-389.

Walters, E. T. (1994). Injury-related behavior and neuronal plasticity: an evolutionary perspective on sensitization, hyperalgesia, and analgesia. Int Rev Neurobiol 36, 325-427.

Walters, E. T., Carew, T. J., and Kandel, E. R. (1981). Associative learning in Aplysia: evidence for conditioned fear in an invertebrate. Science 211, 504-506. 